\title{
Individual and spatio-temporal variations in the home range behaviour of a long-lived, territorial species
}

\author{
Letizia Campioni - María del Mar Delgado • \\ Rui Lourenço · Giulia Bastianelli • Nestor Fernández • \\ Vincenzo Penteriani
}

\begin{abstract}
Despite the fact that investigations of home range behaviour have exponentially evolved on theoretical, analytical and technological grounds, the factors that shape animal home range behaviour still represent an unsolved question and a challenging field of research. However, home range studies have recently begun to be approached under a new integrated conceptual framework, considering home range behaviour as the result of the simultaneous influences of temporal, spatial and individual-level processes, with potential consequences at
\end{abstract}

Electronic supplementary material The online version of this article (doi:10.1007/s00442-012-2493-7) contains supplementary material, which is available to authorized users.

L. Campioni $(\varangle) \cdot$ M. M. Delgado $\cdot$ R. Lourenço .

N. Fernández $\cdot$ V. Penteriani $(\bowtie)$

Department of Conservation Biology, Estación Biológica de Doñana, C.S.I.C., c/Americo Vespucio s/n, 41092 Seville, Spain e-mail: letizia@ebd.csic.es

V. Penteriani

e-mail: penteriani@ebd.csic.es

M. M. Delgado

Metapopulation Research Group, Department of Biosciences, University of Helsinki, 00014 Helsinki, Finland

\section{R. Lourenço}

LabOr, Laboratory of Ornithology and Institute of Mediterranean Agricultural and Environmental Sciences, University of Évora, 7002-554 Évora, Portugal

G. Bastianelli

Department of Biology and Biotechnology "Charles Darwin", University "La Sapienza", P.le A. Moro, 5, 00185 Rome, Italy

V. Penteriani

Finnish Museum of Natural History, Zoological Museum,

University of Helsinki, 00014 Helsinki, Finland the population level. Following an integrated approach, we studied the influence of both external and internal factors on variations in the home range behaviour of 34 radiotagged eagle owl (Bubo bubo) breeders. Home range behaviour was characterised through complementary analysis of space use, movement patterns and rhythms of activity at multiple spatiotemporal scales. The effects of the different phases of the biological cycle became considerably evident at the level of movement patterns, with males travelling longer distances than females during incubation and nestling periods. Both external (i.e. habitat structure and composition) and internal (i.e. sex and health state) factors explained a substantial amount of the variation in home range behaviour. At the broader temporal scale, home range and core area size were negatively correlated with landscape heterogeneity. Males showed (1) smaller home range and core area sizes, (2) more complex home range internal structure and (3) higher rates of movement. The better the physiological condition of the individuals, the simpler the internal home range structure. Finally, inter- and intra-individual effects contributed to shaping space use and movement patterns during the biological cycle. Because of the plurality of behavioural and ecological processes simultaneously involved in home range behaviour, we claim that an integrative approach is required for adequate investigation of its temporal and spatial variation.

Keywords Spatial ecology - Animal movement . Behavioural consistency $\cdot$ Bubo bubo $\cdot$ Homogeneous environment

\section{Introduction}

From early observations regarding the fundamental characteristics of animal movement within a limited space 
(“...most animals and plants keep to their proper home", Darwin 1861) to the advent of sophisticated telemetry technology, such as GPS devices (Cagnacci et al. 2010), for tracking animal movements in challenging environments and conditions, the study of home range behaviour has exponentially evolved on theoretical, analytical and technological grounds, which have now diverged into separate lines of research (Börger et al. 2008; Kie et al. 2010; Smouse et al. 2010). However, the factors that shape animal home range behaviour (i.e. restricted movements in finite areas) still represent an unsolved question and a challenging field of research (Hays 2008). Home range behaviour is comprised of complex and dynamic patterns of space use resulting from routine activities associated with basic aspects of species life-histories (Börger et al. 2006). While the intrinsic complexity of home range behaviour and its consequential spatial expression (i.e. home range patterns) could be the result of potential influences of both internal (e.g. body condition, reproductive status) and external (e.g. landscape structure and composition, food availability) factors, the dynamic nature of home range behaviour may be the consequence of temporal changes of internal and external factors during an individual's lifetime (Börger et al. 2008). Specifically, changes in the internal state of individuals may determine the specific time allocated to different behaviours (e.g. food acquisition, predator avoidance and landscape exploration), thus affecting the properties of the resultant home range patterns. Accordingly, the time allocated to different behaviours may have relevant consequences at both the individual and population levels through modulating survival, reproduction and, as an end result, population dynamics (Morales et al. 2010).

Recently, home range behaviour has begun to be approached from a new perspective intended to redirect previous disconnected areas of knowledge to an integrated conceptual framework that recognises home range behaviour as the result of the simultaneous influences of temporal, spatial and individual-level processes (Börger et al. 2006, 2008; Horne et al. 2008; Indermaur et al. 2009; van Beest et al. 2011). This integrative approach is particularly needed when considering that different home range patterns can emerge from multiple spatio-temporal scales (e.g. McLoughlin and Ferguson 2000; Anderson et al. 2005; van Beest et al. 2011). Following this integrated approach, we investigated here the influence of both external and internal factors on the variations of home range behaviour in a long-lived, territorial (i.e. the same home range is expected to be occupied over many years) nocturnal raptor, the eagle owl (Bubo bubo), during different phases of its biological cycle and at different spatial scales. We expected to detect the concurrent action of three main factors: (1) the effect of the individual changing needs during different periods of the year: because each period of the biological cycle entails specific tasks (e.g. territorial and sexual displays, feeding of young), the home range behaviour is expected to change over the year; (2) the physical characteristics of the nest site surroundings: because the structure and composition of the home range environment and the availability of the main trophic resources have been shown to represent some of the key factors determining differences in the owner's behaviours (Saïd et al. 2009; Rivrud et al. 2010), it is also to be expected that individuals inhabiting areas characterised by different environmental conditions should show different behaviours and home range structures; (3) the characteristics of the home range owners: if variations in home range behaviours are not only based on external factors but also on the intrinsic characteristics of the breeders (i.e. their sex and physiological condition), then we should expect that behaviours and home range features will also be influenced by individual heterogeneity. Shifting from a more general to a species-specific perspective, we may also expect that (1) males should present higher activity rates than females because they perform most of the territorial displays and that the home ranges and core areas of males should increase during the incubation and nestling periods, when males have to find food for both females and chicks, and successively decrease during the post-fledging dependence period, when breeders frequently follow fledglings during their relatively short displacements around the nest (Delgado et al. 2009); (2) habitat heterogeneity and high food availability should decrease both home range sizes and daily movements; and (3) because of their extremely territorial behaviour, males should exhibit smaller home ranges than females, as females are allowed to intrude into the territories of neighbouring pairs with less conflict (Penteriani et al. 2007a).

\section{Materials and methods}

\section{Data collection}

This study was conducted in a hilly area of the Sierra Norte of Seville (Sierra Morena massif) located in south-western Spain. From 2004 to 2010, 34 breeding individuals (24 males and 10 females) from 24 nests were radiotracked. Each individual was fitted with a 30 -g radio-transmitter using a Teflon ribbon backpack harness (Biotrack; Wareham, Dorset, UK; http://www.biotrack.co.uk). The mass of the backpack was less than $3 \%$ of the mass of the smallest adult male $(1,550 \mathrm{~g})$ in our population (mean \pm $\mathrm{SE}=1,667 \pm 105 \mathrm{~g})$. The transmitters included a mercury posture sensor that allowed us to record individual activity (roosting vs. movement) through changes in the 
frequency of the signal (Penteriani et al. 2008). We trapped breeding individuals using two methods: (1) simulating an intrusion with a taxidermic mount and playback of a male call (see Penteriani et al. 2010 for more details), during which a net behind the mount caught responding individuals that only included males because this is the sex that generally engages in aggressive interactions towards intruders (Penteriani et al. 2007a); and (2) using a bow-net (Northwoods, Rainier, WA, USA) placed in the nest when nestlings were 20-35 days old (i.e. when they were already able to thermoregulate). Specifically, nestlings were moved to a box with a metal grid, making them visible to their parents, which were caught when they returned to the nest. After each bow-net trapping session (which lasted from sunset to sunrise), we fed the nestlings and released them into the nest. The individual manipulation was always safe: during 7 years of trapping and continuous radiotracking of breeders (and $>100$ dispersing individuals), we never recorded a potential adverse effect of the backpacks on bird survival or on breeding performance.

We followed territory holders individually throughout the night (from $1 \mathrm{~h}$ before sunset to $1 \mathrm{~h}$ after sunrise; total time duration $=3,333 \mathrm{~h}$ ) during 296 continuous radiotracking sessions (mean number of radiotracking session per owl $\pm \mathrm{SD}=10 \pm 6$ ). We recorded a new location (total number of locations $=5,298$ ) each time we detected a change in the position of the focal individual (for more detail on movement detections, see Penteriani et al. 2008; mean number of locations per radiotracking session $\pm \mathrm{SD}=17.2 \pm 5.2$ ). Therefore, the number of recorded locations represented the effective number of movements for an individual during each night. The continuous radiotracking sessions (mean time duration of a radiotracking session $\pm \mathrm{SD}=11.3 \pm 1.8 \mathrm{~h}$ ) were performed year-round in an attempt to obtain an homogenous dataset over the different phases of the owl's biological cycle until either the individual died or the battery of the transmitter ran out (lifespan of transmitters from $\sim 1.5$ to $\sim 2.5$ years). The locations of radiotagged individuals were determined by triangulations using three-element hand-held Yagi antennae (Biotrack) with Stabo (XR-100) portable ICOM receivers (IC-R20). Triangulations were generally performed within a small range of distances of the focal owl (100-300 m), with an accuracy of $83.5 \pm 49.5 \mathrm{~m}$ (mean $\pm \mathrm{SE}$ ) (Penteriani and Delgado 2008a). This value was estimated in cases when, after a fix, we needed to locate an individual to manipulate it during field experiments (Penteriani et al. 2007b) or to record the cause of mortality when the individual died. The tracking did not appear to affect the behaviour of the owls, which generally appeared to ignore the observer (Delgado et al. 2009).
Characterising home range behaviour

\section{Space use}

Space use was studied at two different temporal scales. First, the seasonal scale relies on the biological cycle of the species, i.e. the pre-laying (September-mid-January, period 1), incubation (mid-January-mid-March, period 2), nestling (mid-March-early April, period 3) and fledgling/postfledging dependence (F/PFD) (early April-August, period 4; see: Delgado and Penteriani 2007) periods. Second, the overall scale encompassed the entire period during which we were able to follow an individual (mean number of months during which each radiotagged owl was followed $\pm \mathrm{SD}=15 \pm 8$; range $5-33$ ). That is, this scale is not linked to the biological cycle and it has been only used to describe general patterns (i.e. global home range and core area sizes, core area-nest distance).

Working at two different spatial scales (home range and core area), we quantified the space use of tagged individuals using four descriptors. We first estimated home range size through fixed-kernel methods (Worton 1989) using the Animal Movement Extension for ArcView 3.2 (Hooge and Eichenlaub 2000). We calculated the 50 and $90 \%$ fixed kernels using the least squares cross-validation (LSCV) procedure (Silverman 1986) to determine the optimal value of the smoothing parameter for a given kernel and sample size (Seaman et al. 1999). The LSCV process generates the best value of the smoothing parameter for multimodal data with respect to the other methods (Silverman 1986; Worton 1989; Seaman and Powell 1996). We chose the $50 \%$ kernel to represent the core areas after a detailed exploratory analysis because it allows (1) including a sufficient number of locations and (2) comparisons with similar studies. To establish home range boundaries, we preferred to use density isopleth values of $90 \%$ because this value fitted better with our data, giving more accurate estimates when analysing more than 30 relocations. In fact, when visually exploring both 90 and $95 \%$ isopleths, the density isopleth values of $95 \%$ over-estimated the areas crossed by tagged individuals. We used all data available for each individual, focusing more on the biological process that shaped home range internal structure (De Solla et al. 1999) than on obtaining statistical independence of the relocations. This was possible because we followed each focal owl during the entire period of its nocturnal activity, thus recording its entire set of movements. Finally, because individual variation in the number of relocations may potentially contribute to variability in estimates of space use (Kernohan et al. 2001), we regressed the number of relocations with home range size, but no relationships were found $\left(r^{2}=0.008 ; F_{1,25}=0.19 ; P=0.67\right)$. 
Second, with the aim of characterising the internal structure of each home range, we estimated the size of core area(s), i.e. the areas most frequently used within the home range. Because it was not always possible to distinguish between the core area of the nest and the core area(s) where individuals repeatedly hunted every night, in the present study, core area(s) represented both nesting and hunting areas. Again, when regressing the number of relocations with core area size, we did not find any relationship $\left(r^{2}=0.008 ; \quad F_{1,25}=0.81 ; \quad P=0.38\right)$. Third, we also estimated the number of core areas per home range as a measure of the amount of most frequently visited sites, and fourth, the distance between the exact location of the nest and the geometrical centre of each core area.

\section{Movement patterns}

Individual nightly movement behaviour was characterised by five variables: (1) total distance, as the sum of the distance between successive steps of the nightly displacements; (2) step length, as the distance between successive locations; (3) speed, as the step length divided by the time interval between successive locations; (4) turning angle between successive movements; and (5) time step, as the time elapsed between successive moves. The movement variables were analysed at two different spatial scales, home range and core area, and two temporal scales, overall and seasonal.

\section{Rhythms of activity}

The nocturnal activity of tagged owls was estimated using two indices: (1) core area activity, i.e. the percentage of time an owl spent inside the core area(s); and (2) individual movement rates, calculated as the movement frequencies (a) per night and (b) within the core areas. Core area activity is a measure of the time devoted to main activities, such as hunting, feeding (including nestling/fledgling feeding and female feeding during breeding if the focal owl was a male) and territorial defence. Because night lengths vary year round, we standardised the core area activities and movement rates per night by dividing them by the total time the owl was active each night; movement rates within core areas were standardised to account for the total amount of movements performed by the focal owl per night.

Laying dates and breeding success

During the entire study period, for each of the 24 nests where we trapped breeders, we recorded (1) the egg laying date and (2) the number of fledglings. Egg laying dates were determined by estimating the age of nestlings following Penteriani et al. (2005) and assuming 33 days of incubation. Both the egg laying date and number of fledglings were used as response variables to detect potential effects of home range behaviour and internal and external factors on breeding phenology and success.

Individual characteristics and internal state

We correlated space use, movement patterns, rhythms of activity and breeding success with three characteristics of an individual: (1) sex, determined by molecular procedures using DNA extracted from blood (Griffiths et al. 1998); (2) age, estimated based on the moult pattern of the feathers (Martínez et al. 2002); and (3) haematocrit (HT), as an indicator of physiological condition. HT has been widely used as an indicator of nutritional status because nutritional deficiencies result in anaemia due to shortages in essential amino acids (e.g. Costa and Macedo 2006). To obtain HT values, blood samples were collected and stored in tubes with heparin at $4{ }^{\circ} \mathrm{C}$ until arrival at the laboratory, where they where centrifuged for $10 \mathrm{~min}$ at $4,000 \mathrm{rpm}$.

\section{External factors}

To determine the possible influence of external factors on home range behaviour and breeding success, we estimated three variables (detailed in the following sections: "Eagle owl diet and rabbit census" and "Landscape characteristics of home ranges and core areas"): (1) as diet analyses showed that the rabbit Oryctolagus cuniculus (Linnaeus 1758 ) is the main prey of our eagle owl population (mean biomass percentage of rabbit in the diet $=62.0 \pm 19.1 \%$, range 16-94\%) and given the distribution overlap of both species (Delibes-Mateos et al. 2007), we considered rabbit abundance within the home ranges of tagged individuals as an indicator of habitat quality (González et al. 2008); (2) the contribution of rabbits (\% of biomass) to the diet of breeders; and (3) landscape characteristics of home ranges and core areas.

\section{Eagle owl diet and rabbit census}

The diet of eagle owls was determined by analysing prey remains and pellets collected from 2003 to 2008 during visits to nests and roosting and feeding perches at 24 nest sites. Following Lourenço (2006), we identified 4,203 prey items using identification keys for bones and feathers and comparisons with a reference collection (Laboratory of Archaeo-sciences, IGESPAR, Portugal), followed by determining the minimum number of individuals. When possible, prey items were identified to the species taxonomic level. We calculated the biomass percentage for each prey species using its mean weight value from 
bibliographic references or bone measurements to estimate the weight of each individual (Cramp and Simmons 1977-1994; Donázar and Ceballos 1989; MacDonald and Barret 1993).

The relative rabbit abundance was estimated in the 24 breeding areas using rabbit faecal pellet counts (i.e. latrine counts). Latrine counts have been previously used as an index to estimate rabbit abundance (Palma et al. 1999) and are a good indirect estimator of rabbit abundance in largescale studies (Palomares 2001a, b; Fernández 2005). The census was conducted in 2009 from the beginning of March to the beginning of May. This period corresponds to the nestling and F/PFD phases of eagle owls, when it is expected that parents exhibit the highest hunting effort. To obtain comparable indices of prey abundance (IKA) for each territory and around each nest, we drew a circular plot with an area equal to the mean eagle owl home range size in our study population, which was calculated using the minimum convex polygon method (MPC; Hayne 1949). Inside these plots, we walked $2.2-\mathrm{km}$-long transect lines, recording the number of latrines found on both sides of each transect within a 4-m width. Latrine counts were always performed by the same observers (walking at the speed of $1 \mathrm{~km} \mathrm{~h}^{-1}$ ), and the IKA was expressed as the number of latrines per $\mathrm{km}$ of transect; the total length of transects walked was $150 \mathrm{~km}$, in which we counted 3,440 latrines (mean \pm SE $20.6 \pm 12.4 \mathrm{~km}^{-1}$, range 7.7-46.0 $\mathrm{km}^{-1}$ ). Rabbit density over the years can be considered relatively stable in our study area: rabbit management and frequent releases inside our study area have created extremely favourable and steady trophic conditions (Penteriani and Delgado, unpublished data).

\section{Landscape characteristics of home ranges and core areas}

We measured landscape characteristics by intersecting a digital layer representing the boundaries of the owl's home ranges and core areas with a map of landcover elements (scale 1:25,000, Junta de Andalucia, Consejería de Medio Ambiente, 2003). Landscape composition was analysed at the two spatial scales previously used in the analyses of home range behaviour. Following Aebischer et al. (1993), with the aim of selecting only those habitat types that were most relevant for eagle owls, we (1) first performed a compositional analysis to test owl habitat selection and then (2) classified the landscape at the two different spatial scales. At the fine-grained spatial scale of analysis (i.e. the core area), landscape composition was represented by ten landcover types: urban areas, water bodies, forests, dense scrublands with trees, sparse scrub with trees, herbaceous vegetation with trees, scrublands, low vegetation, woody crops and herbaceous. For the coarse-grained scale (i.e. the home range), landscape composition was simplified into six categories: urban/crops areas, water bodies, dense vegetation (forest and dense scrubs with trees), sparse scrub with trees, herbaceous areas with and without trees, and scrublands. These habitat types were then employed to model the variation in individual home range behaviour. Additionally, we used edge density (i.e. the total length of the patch edge per unit area within each landscape; Elkie et al. 1999) as a proxy for the effect of habitat heterogeneity (Donovan et al. 1995; Kie et al. 2002; Anderson et al. 2005). The GIS application ArcView 3.2 and its extension Patch Analyst (Elkie et al. 1999) were used for the analyses of landscape characteristics.

\section{Data analysis}

We constructed a set of a priori competing models starting from the simplest null model (intercept only model) to a full model that included all of the explanatory variables (see Tables S1-S4 in Online Resource for fitted variables).

First, we conducted a graphical analysis for the entire set of explanatory variables and checked for correlations (Spearman's rank correlation) among predictors, excluding variables with $r_{\mathrm{s}} \geq 0.6$. For each analysis, we used different subsamples, represented by those owls for which it was possible to obtain the required information. Thus, in each analysis, the type and number of explanatory variables were selected on the basis of their biological relevance, our interest and sample sizes. Because we had repeated measures for the same individual within and between years, we included individual identity (ID), together with nest site nested in year as first-, second- and third-level random effects, respectively. Following Pinheiro and Bates (2004), the significance values of random effects were estimated using the Akaike information criterion (AIC). When random factors did not improve the model's likelihood value, we built a less complex model class. The models were checked for unequal variance structures of the withingroup errors by investigating relevant model diagnostic plots (plots of residuals vs. fitted values for the relevant model and variable; Pinheiro and Bates 2004) and by comparing models with and without different variance functions using the AIC. If selected, following Pinheiro et al. (2009), we implemented variance functions in the models. We also checked for any remaining dependencies among the within-group errors after the fixed and random effects were fitted. If present, these were modelled using correlation structures. The spatial autocorrelation between home ranges and core areas was corrected using the mean coordinates of each home range and core area, while movement variables were corrected using the spatial coordinates of each fix (UTM coordinates). In all cases, different correlation structures were specified, and, if necessary, the most appropriate was selected by comparing the 
AIC values of the fitted models (see Pinheiro and Bates 2004). Detection of the most parsimonious hypothesis was based on (1) model selection procedures using the AIC, which allows the comparing of multiple working hypotheses and weighting their level of support in the data, or (2) a second-order AIC derivation, the AICc, which is appropriate when the ratio of the sample size to the number of parameters is less than 40 (Burnham and Anderson 2002). Two AIC statistics were also calculated for each model: $\triangle \mathrm{AIC}$ and $\mathrm{AICw}_{i}$, which indicate the probability that the model selected is the best among the different candidates. Values of $\Delta$ AIC $\leq 2$ were used as the criterion for selecting the best models, i.e. those with substantial support from the data (Burnham and Anderson 2002). For simple linear models (LMs) and general linear models (GLMs and GLMMs), we also reported $r^{2}$ and deviance values, respectively. For models including random terms, we presented (1) the intraclass correlation coefficient (hereafter ICC, see Zuur et al. 2009), which is a measure of the correlation between observations from the same group (i.e. owl ID) and is expressed as ICC $=d^{2} / d^{2}+\sigma^{2}$, where $d^{2}$ is the covariance between any two observations for the same individual and its variance is $d^{2}+\sigma^{2}$; and (2) a generalised $R^{2}$ for random effect, which provides information about the amount of variation in the data explained by the random effect (i.e. between-individual variation). This parameter was calculated as the squared correlation between the fitted values of the model and the observed values in the data (Zheng and Agresti 2000). Sex was a relevant factor in almost all analyses, but the small sample size of some subsamples did not allow the testing for interactions; therefore, we divided the database into two different subsets: one for males and one for females. Because females rest motionless in the nest during most of the incubation period, no data were available to make inter-gender comparisons in this period. Values are given as the mean \pm SD and range. All analyses were performed using the R software package ( $\mathrm{R}$ Development Core Team 2009). The following specific $\mathrm{R}$ functions were performed: (1) adehabitat $1 \cdot 8 \cdot 3$, for compositional analysis (Calenge 2006); (2) nlme 3.1-92 (Pinheiro et al. 2009), for linear multilevel mixed-effects models (LMMs), as described by Pinheiro and Bates (2004); and (3) the lme4 0.999375-28 package for GLMM (Bates and Sarkar 2007) and multcomp (Hothorn et al. 2009) for multiple comparisons.

\section{Post hoc test for seasonal effects}

To obtain additional insights regarding seasonal variations in the owls' space use, movement patterns and rhythms of activity, we used Simultaneous Tests for General Linear Hypotheses, in which multiple comparisons of means were performed using the Tukey Contrasts method (Hothorn et al.
2009). This type of post hoc test allows for the detecting of differences among all factor levels: in our case, there were four factor levels corresponding to the different phases of the eagle owl biological cycle. Differences among levels were considered significant at $P<0.05$.

\section{Characterising home range behaviour}

\section{Space use}

Depending on the nature of the response variables and the presence or absence of random effects, we fitted a suite of different models: (1) LMs, for log-transformed home range size, core area size and core area-nest distance at the overall timescale; (2) LMMs, for the same log-transformed response variables cited above (but at the seasonal timescale), including individual identity (i.e. owl ID) as a random effect; and (3) general linear models (GLMs) for the number of core areas at the overall and seasonal timescales. Because the number of core areas could be 1 or $>1$, this response variable was modelled using a binomial distribution $(0=>1$ core area; $1=1$ core area $)$.

\section{Movement patterns}

Some variables describing movement patterns (total distance, step length, time step and speed) were $\log +1$-transformed and modelled using LMMs at both the home range and core area spatial scales. We always included individual identity as a random effect at the home range spatial scale when fitting the LMMs. Additionally, temporal autocorrelation (using the corExp function in the R library nlme) was included when fitting the step length and speed models, while a variance structure (using the varPower function with the year as a covariate) was used to model the time step. Turning angles were simplified into an index of 1 for positive and 0 for negative angle cosine values (forward and backward movements, respectively) and modelled using a GLM with a binomial distribution. At the core area spatial scale, the entire models included individual identity as a random effect, and the time step and speed models also included year, as a second-level random effects. A temporal autocorrelation (using the corExp function) was fitted to improve the step length, time step and speed models. Finally, at this spatial scale, turning angle was modelled using a GLMM.

\section{Rhythms of activity}

While at the core area spatial scale, core area activity was $\log +1$-transformed and modelled together with movement rate using LMs; at the home range scale, movement rate was $\log +1$-transformed and modelled using an LMM, in which year was specified as a random effect. 
Breeding phenology and breeding success

For laying date, we fitted a LMM that included individual identity and nest site as first- and second-level nested random effects. Similarly, the mean number of fledglings was modelled using LM (see Table S4 in Online Resource).

\section{Results}

Characterising home range behaviour

\section{Space use}

The home ranges of females were larger and showed higher inter-individual variation than the home ranges of males (Table 1); the size of core areas for females was also larger than for males, although the variation in core area size was consistently similar between sexes (Table 1). The size variations of home range and core areas at the overall timescale (Table 1; Fig. 1) were partially explained by two models ( $r^{2}=0.23$ and 0.37 , respectively) that included the same factors, i.e. edge density (home range model estimates \pm $\mathrm{SE}=-0.006 \pm 0.003$; core area $=-0.007 \pm 0.002$ ) and sex (home range model estimates $\pm \mathrm{SE}=-0.596 \pm 0.299$; core area $=-0.333 \pm 0.373 ;$ Fig. S1 in Online Resource). A second competing model for core area only included edge density as the unique explanatory variable (Table S1 in Online Resource; Fig. 1). That is, an increase in the amount of edge density, which is a proxy of habitat heterogeneity, at both spatial scales resulted in a decrease of the home range and core area sizes (Table S1 in Online Resource).

With respect to home range and core area size variations at the seasonal timescale (Table S1 in Online Resource), no single model was strongly supported as approximating home range and core area variations (Table 1; Fig. S2 in Online Resource). Additionally, an ICC value (for the owl ID random term) of 0.63 supported this consistency in home range size during each period of the biological cycle. Although, in the core area analysis, the model that included sex as a predictor ranked as the second best model, the set of the most supported models included the null model, and, thus, it was not possible to distinguish the most plausible model. In summary, our seasonal analyses did not find relevant differences in home range behaviour between sexes or among the four periods of the owl biological cycle. This result was confirmed by post hoc analysis (always $P>0.05$ ). Conversely, we observed high betweenindividual variation in home range size, as shown by the $R^{2}$ value for the random term of 0.71 (Table $\mathrm{S} 1$ in Online Resource).

When analysing the internal structure of the home ranges at the overall timescale (Table S1 in Online Resource), we first found that the better the physiological condition of the individuals (i.e. the higher the HT values; $50.28 \pm 1.52 \%$ ), the simpler the internal structure of their home range, i.e. closer to the nest (model estimate $\pm \mathrm{SE}=-0.053 \pm 0.018$; $r^{2}=0.26$; Fig. 1) and a smaller number of core areas (model estimate $\pm \mathrm{SE}=0.207 \pm 0.111$; deviance $=0.29$ ). Additionally, males exhibited a slightly greater number of core areas than females (Table 1); the core areas of males were located at greater distances from the nest than those of females (Table 1). However, these models only captured a relatively low amount of the variation in the data.

Regarding our analysis of the internal structure of the home ranges at the seasonal scale (Table S1 in Online Resource), the owls did not show any variation in the internal structure of their home ranges among different phases (Table 1). Finally, none of the models was supported regarding core area-nest distances at the seasonal timescale (Table 1 and Table $\mathrm{S} 1$ in Online Resource).

\section{Movement patterns}

At the home range spatial scale, the period of the biological cycle slightly affected both total distance (intercept + period 1 model estimate $\pm \mathrm{SE}=8.757 \pm 0.060 ;$ period $2=$ $0.228 \pm 0.078 ;$ period $3=0.134 \pm 0.099 ;$ period $4=$ $-0.121 \pm 0.060$; deviance $=0.32$ ) and step length (intercept + period 1 model estimate $\pm \mathrm{SE}=5.661 \pm 0.050$; period $2=0.191 \pm 0.048$; period $3=0.086 \pm 0.064$; per$\operatorname{iod} 4=0.016 \pm 0.039 ;$ deviance $=0.06$; Table $1 ;$ Table S2 in Online Resource). Again, as was found for home range and core area size variations at the seasonal timescale, the between-individual variation $\left(R^{2}=0.25\right)$ and consistency $(\mathrm{ICC}=0.21)$ regarding total distance were considerable. Individuals travelled longer distances during incubation and nestling periods than during pre-laying and F/PFD periods (Table 1). Differences in speed could not be discerned because the intercept only model was included as the best supported model. Finally, the best models for turning angle and time step (Table 1) included the combination of two vegetation types (turning angle: dense vegetation model estimate $\pm \mathrm{SE}=0.004 \pm 0.001 ;$ sparse scrub: $0.004 \pm$ 0.002 ; deviance $=0.01)$ and age (model estimate $\pm \mathrm{SE}=$ $0.082 \pm 0.021 ;$ deviance $=0.03$ ), respectively (Table 1 ; Table S2 in Online Resource). However, very little variation was associated with these explanatory variables.

At the finer core area spatial scale, speed and turning angle were not related to any of the considered external or internal factors (Table 1). Similarly, despite the fact that step length and time step were sex-dependent, with females travelling in shorter steps at longer time intervals than males (Table 1; Table $\mathrm{S} 2$ in Online resources), the null models always ranked as the most parsimonious for all 
Table 1 Descriptors (mean \pm SD, range and sample size) of eagle owl (Bubo bubo) home range behaviours

\begin{tabular}{|c|c|c|c|c|c|c|c|}
\hline \multirow[t]{3}{*}{ Scale } & \multicolumn{4}{|l|}{ Space use } & \multicolumn{3}{|c|}{ Rhythms of activity } \\
\hline & \multicolumn{2}{|l|}{ Home range } & \multicolumn{2}{|l|}{ Core area } & \multirow{2}{*}{$\begin{array}{l}\text { Home range } \\
\text { Movement } \\
\text { rate }^{\mathrm{a}}\end{array}$} & \multicolumn{2}{|l|}{ Core area } \\
\hline & $\begin{array}{l}\text { Home range } \\
\text { size (ha) }\end{array}$ & $\begin{array}{l}\text { Core area } \\
\text { size (ha) }\end{array}$ & $\begin{array}{l}\text { Core area } \\
\text { number }\end{array}$ & $\begin{array}{l}\text { Nest-core area } \\
\text { distance }(\mathrm{m})\end{array}$ & & $\begin{array}{l}\text { Movement } \\
\text { rate }^{\text {b }}\end{array}$ & Activity $^{\mathrm{c}}$ \\
\hline \multicolumn{8}{|l|}{ Overall } \\
\hline \multirow[t]{3}{*}{ Male } & $187.1 \pm 28.8$ & $34.1 \pm 6.8$ & $1.5 \pm 0.2$ & $486 \pm 64.4$ & $0.028 \pm 0.001$ & $0.27 \pm 0.01$ & $0.38 \pm 0.02$ \\
\hline & $283.7-548.4$ & $4.4-104.4$ & $1-3$ & $76-869$ & $0.011-0.042$ & $0-1$ & $0-1$ \\
\hline & 20 & 19 & 19 & 19 & 216 & 176 & 220 \\
\hline \multirow[t]{3}{*}{ Female } & $309.7 \pm 85.4$ & $56.3 \pm 18.5$ & $1.2 \pm 0.2$ & $287 \pm 99.6$ & $0.025 \pm 0.001$ & $0.35 \pm 0.05$ & $0.35 \pm 0.05$ \\
\hline & $121.3-695.0$ & $12.3-123.4$ & $1-3$ & $140-731$ & $0.014-10.042$ & $0-1$ & $0-1$ \\
\hline & 7 & 7 & 7 & 6 & 40 & 29 & 40 \\
\hline \multirow[t]{3}{*}{ All } & $218.9 \pm 30.8$ & $40.1 \pm 6.9$ & $1.4 \pm 0.1$ & $438.2 \pm 55.5$ & $0.028 \pm 0.0004$ & $0.29 \pm 0.01$ & $0.37 \pm 0.02$ \\
\hline & $283.7-695.0$ & $4.4-123.4$ & $1-3$ & $76-869$ & $0.011-0.047$ & $0-1$ & $0-1$ \\
\hline & 27 & 26 & 26 & 25 & 256 & 205 & 260 \\
\hline \multicolumn{8}{|l|}{ Seasonal } \\
\hline \multicolumn{8}{|c|}{ Pre-laying } \\
\hline \multirow[t]{3}{*}{ All } & $149.0 \pm 17.7$ & $26.8 \pm 3.9$ & $1.6 \pm 0.2$ & $485.7 \pm 80.7$ & $0.026 \pm 0.001$ & $0.28 \pm 0.03$ & $0.42 \pm 0.03$ \\
\hline & $23.1-324.1$ & $3.6-589.7$ & $1-3$ & $137-1,504$ & $0.013-0.046$ & $0-1$ & $0-1$ \\
\hline & 21 & 21 & 21 & 21 & 81 & 68 & 75 \\
\hline \multicolumn{8}{|c|}{ Incubation } \\
\hline \multirow[t]{3}{*}{ Male } & $156.7 \pm 25.3$ & $32.7 \pm 6.4$ & $1.3 \pm 0.2$ & $522.1 \pm 132.4$ & $0.028 \pm 0.001$ & $0.30 \pm 0.03$ & $0.45 \pm 0.04$ \\
\hline & $28.4-340.1$ & $41.0-760.3$ & $1-3$ & $48-1,720$ & $0.018-0.044$ & $0-1$ & $0-1$ \\
\hline & 15 & 14 & 16 & 15 & 44 & 37 & 41 \\
\hline \multicolumn{8}{|c|}{ Nestling } \\
\hline \multirow[t]{3}{*}{ All } & $136.3 \pm 23.3$ & $21.6 \pm 5.3$ & $1.9 \pm 0.4$ & $509.9 \pm 109.6$ & $0.029 \pm 0.001$ & $0.30 \pm 0.06$ & $0.44 \pm 0.07$ \\
\hline & $57.9-225.0$ & $53.290-462.6$ & $1-3$ & $201-967$ & $0.017-10.047$ & $0-1$ & $0-1$ \\
\hline & 8 & 8 & 8 & 8 & 21 & 15 & 17 \\
\hline \multicolumn{8}{|l|}{ F/PFD } \\
\hline \multirow[t]{3}{*}{ All } & $218.0 \pm 5.4$ & $36.3 \pm 6.3$ & $1.6 \pm 0.2$ & $504.4 \pm 68.7$ & $0.028 \pm 0.001$ & $0.30 \pm 0.02$ & $0.39 \pm 0.03$ \\
\hline & $39.4-570.0$ & $52.2-940.2$ & $1-3$ & $80-1,140$ & $0.011-0.047$ & $0-1$ & $0-1$ \\
\hline & 20 & 20 & 22 & 20 & 114 & 85 & 103 \\
\hline
\end{tabular}

Scale Movement patterns

\begin{tabular}{|c|c|c|c|c|c|c|c|c|}
\hline \multicolumn{5}{|c|}{ Home range scale } & \multicolumn{4}{|c|}{ Core area scale } \\
\hline $\begin{array}{l}\text { Total } \\
\text { distance } \\
\text { (m) }\end{array}$ & $\begin{array}{l}\text { Step } \\
\text { length } \\
(\mathrm{m})\end{array}$ & $\begin{array}{l}\text { Speed } \\
(\mathrm{m} / \mathrm{min})\end{array}$ & $\begin{array}{l}\text { Time } \\
\text { step } \\
\text { (min) }\end{array}$ & $\begin{array}{l}\text { Cos } \\
\text { (turning } \\
\text { angle) }\end{array}$ & $\begin{array}{l}\text { Step } \\
\text { length } \\
\text { (m) }\end{array}$ & $\begin{array}{l}\text { Speed } \\
(\mathrm{m} / \mathrm{min})\end{array}$ & $\begin{array}{l}\text { Time } \\
\text { step } \\
(\mathrm{min})\end{array}$ & $\begin{array}{l}\text { Cos } \\
\text { (turning } \\
\text { angle) }\end{array}$ \\
\hline
\end{tabular}

Overall

Male

$33.5 \pm 0.51$

$0.03 \pm 0.01$

$244.1 \pm 7.2$

$18.5 \pm 1.4 \quad 33.3 \pm 1.3$

$0.02 \pm 0.02$

1,543-16,190

$3.2-2,844$

$0.001-1,199$

1-217

-1 to 1

4-2,096

4,066

4,068

823

0.001-403.8 1-195

-1 to 1

Female

$6,713 \pm 489.8$

$437.8 \pm 15.3 \quad 36.8 \pm 3.7$

$36.8 \pm 1.38$

$0.03 \pm 0.03$

$220.1 \pm 16.5$

800

797

760

1,152-14,350 14.1-2,843

0.07-1,254

1-213

-1 to 1

14-2,075

$21.7 \pm 4.3$

$42.1 \pm 2.4$

$0.01 \pm 0.05$

$46 \quad 705$

All

$6,322 \pm 187.4 \quad 418.0 \pm 5.2$

$33.0+1.1$

689

689

184

0.07-397.9

1-195

-1 to 1

1,701-16,190 3.2-28,440

$0.001-1,254.0$

$34.0 \pm 0.5$

$224.5 \pm 6.64$

$19.0 \pm 1.4$

181

$277 \quad 4,767$

4,757

4,756

4,757

$4-2,096$

$0.001-403.8$

975

978

$0.02 \pm 0.02$

Seasonal

Pre-laying

\begin{tabular}{|c|c|c|c|c|c|c|c|c|c|}
\hline \multirow[t]{3}{*}{ All } & $6,912 \pm 320.4$ & $396.2 \pm 8.1$ & $31.9 \pm 1.71$ & $36.35 \pm 0.89$ & $0.03 \pm 0.01$ & $231.0 \pm 10.7$ & $21.8 \pm 2.8$ & $36.7 \pm 2.0$ & $0.01 \pm 0.04$ \\
\hline & $1,701-16,190$ & $3.2-2,317.0$ & $0.001-1,254$ & $1-213$ & -1 to 1 & $4-1,628$ & $0.001-403.8$ & $1-178$ & -1 to 1 \\
\hline & 88 & 1,632 & 1,632 & 1,651 & 1,631 & 355 & 349 & 350 & 331 \\
\hline
\end{tabular}


Table 1 continued

\begin{tabular}{|c|c|c|c|c|c|c|c|c|c|}
\hline \multirow[t]{3}{*}{ Scale } & \multicolumn{9}{|c|}{ Movement patterns } \\
\hline & \multicolumn{5}{|c|}{ Home range scale } & \multicolumn{4}{|l|}{ Core area scale } \\
\hline & $\begin{array}{l}\text { Total } \\
\text { distance } \\
(\mathrm{m})\end{array}$ & $\begin{array}{l}\text { Step } \\
\text { length } \\
(\mathrm{m})\end{array}$ & $\begin{array}{l}\text { Speed } \\
(\mathrm{m} / \mathrm{min})\end{array}$ & $\begin{array}{l}\text { Time } \\
\text { step } \\
(\min )\end{array}$ & $\begin{array}{l}\text { Cos } \\
\text { (turning } \\
\text { angle) }\end{array}$ & $\begin{array}{l}\text { Step } \\
\text { length } \\
(\mathrm{m})\end{array}$ & $\begin{array}{l}\text { Speed } \\
(\mathrm{m} / \mathrm{min})\end{array}$ & $\begin{array}{l}\text { Time } \\
\text { step } \\
(\mathrm{min})\end{array}$ & $\begin{array}{l}\text { Cos } \\
\text { (turning } \\
\text { angle) }\end{array}$ \\
\hline \multicolumn{10}{|c|}{ Incubation } \\
\hline \multirow[t]{3}{*}{ Male } & $8,573 \pm 514.5$ & $469.0 \pm 13.4$ & $31.6 \pm 1.79$ & $33.82 \pm 1.12$ & $0.03 \pm 0.02$ & $246.2 \pm 18.1$ & $16.1 \pm 1.9$ & $35.6 \pm 2.6$ & $0.07 \pm 0.05$ \\
\hline & $2,983-15,680$ & $6.6-2,844.0$ & $0.001-845.6$ & $1-213$ & -1 to 1 & $4-2,096$ & $0.07-201.4$ & $1-195$ & -1 to 1 \\
\hline & 43 & 865 & 864 & 866 & 868 & 185 & 179 & 181 & 179 \\
\hline \multicolumn{10}{|c|}{ Nestling } \\
\hline \multirow[t]{3}{*}{ All } & $7,077 \pm 489.7$ & $402.7 \pm 15.9$ & $21.9 \pm 1.41$ & $33.17 \pm 1.37$ & $0.04 \pm 0.03$ & $241.1 \pm 23.5$ & $20.2 \pm 6.0$ & $32.2 \pm 2.7$ & $0.04 \pm 0.07$ \\
\hline & $3,637-13,500$ & $4.5-2,310.0$ & $0.001-605.2$ & $1-157$ & -1 to 1 & $4-1,174$ & $0.11-397.9$ & $1-103$ & \\
\hline & 23 & 408 & 405 & 391 & 409 & 88 & 84 & 84 & 79 \\
\hline \multicolumn{10}{|l|}{ F/PFD } \\
\hline \multirow[t]{3}{*}{ All } & $6,168 \pm 275.2$ & $416.7 \pm 8.8$ & $31.9 \pm 1.54$ & $32.87 \pm 0.74$ & $0.03 \pm 0.01$ & $204.0 \pm 10.1$ & $17.6 \pm 2.0$ & $33.5 \pm 1.6$ & $0.01 \pm 0.04$ \\
\hline & $1,792-15,310$ & $5.8-2,843.0$ & $0.001-1,249$ & $1-217$ & -1 to 1 & $6-2,075$ & $0.07-363$ & $1-195$ & -1 to 1 \\
\hline & 123 & 1,862 & 1,844 & 1,848 & 1,849 & 379 & 363 & 363 & 348 \\
\hline
\end{tabular}

F/PFD Fledgling/post-fledging dependence

${ }^{\text {a }}$ Number of movements night duration ${ }^{-1}$

${ }^{\mathrm{b}}$ Movement inside core area total movements ${ }^{-1}$

c Time spent in hunting area night duration ${ }^{-1}$

Fig. 1 a Plot of logtransformed home range size (ha) of eagle owls (Bubo bubo) in relation to edge density $(\mathrm{m} /$ ha) at the overall time scale for males (open circles) and females (filled circles). Lines represent the predicted effect for males (continuous line) and females (dashed line) separately. b Plot of logtransformed core area-nest distance $(\mathrm{m})$ in relation to haematocrit value $(\%)$ at the core area and overall spatiotemporal scale (see the main text for more details) for males (open circles) and females (filled circles). Lines represent the predicted effect for males (continuous line) and females (dashed line) separately
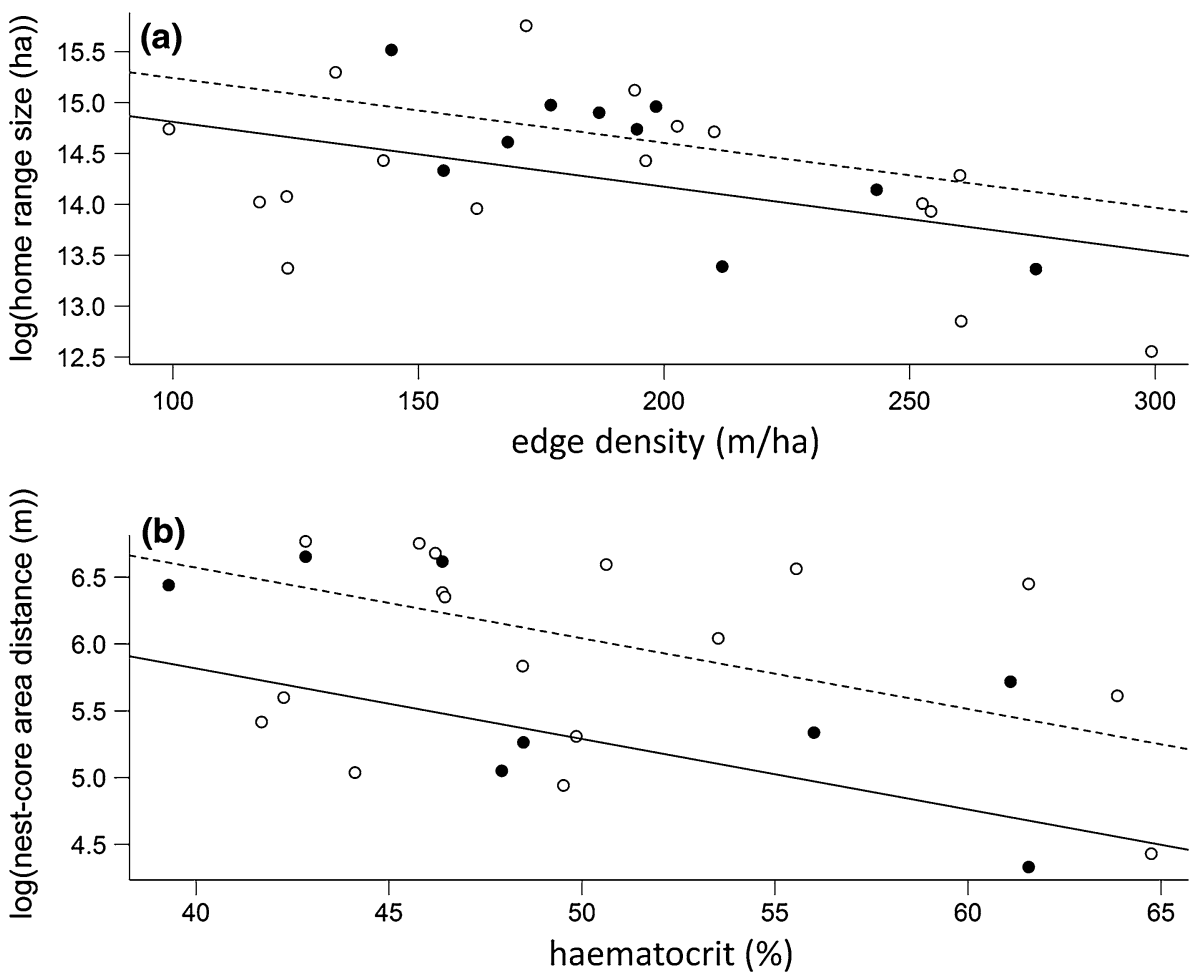

analyses. Moreover, we observed high intra-individual consistency in speed (ICC values for owl ID =0.94) and step length $(\mathrm{ICC}=0.35)$, with a moderate percentage of variance $\left(R^{2}=0.21\right)$ explained by owl ID for step length
(Table S2 in Online Resource). Post hoc analysis, at both the home range and core area spatial scales, showed that males and females presented similar movement behaviour at the seasonal scale ( $P>0.05$ for all periods). 


\section{Rhythms of activity}

The activity patterns of eagle owls $(n=11$ males, $n=6$ females; number of radiotracking nights $=259$ ) were quite constant year round and did not show any clear differences between periods. Movement rate at the home range spatial scale $(0.0276 \pm 0.0004$ number of movements night length $^{-1}, n=256$ ) was not influenced by any external factor. However, we found a little difference between sexes $\left(r^{2}=0.15\right)$, with males (model estimate $\pm \mathrm{SE}=0.005 \pm$ 0.001 ) moving at higher rates than females (model estimate $\pm \mathrm{SE}=0.023 \pm 0.001$; Table 1 ; Table S3 in Online Resource). At the core area spatial scale, the models that included edge density were always the best supported for explaining variation in the owls' movement (edge density model estimate $\pm \mathrm{SE}=-0.0005 \pm 0.0003$; deviance $=$ $0.04)$ and activity rates (edge density model estimate \pm $\mathrm{SE}=-0.0004 \pm 0.0002$; deviance $=0.02$; Table $\mathrm{S} 3$ in Online Resource), with individuals showing higher movement and activity rates when the density of edges decreased. Again, these models only captured a relatively low amount of the recorded patterns.

Laying dates and breeding success

Laying dates ranged from 24 December to 8 April, while the mean number of fledgling chicks was $2.18 \pm 1.03$ (range 1-4 chicks). Although none of the factors considered seem to affect owl laying dates (Table S4 in Online Resource), variation in the number of fledglings was better explained by two univariate competing models (Table S4 in Online Resource): the pairs successfully rearing the highest number of fledglings were those (1) with widest core areas $\left(r^{2}=0.15\right)$ and (2) that consumed the highest $\%$ of rabbits $\left(r^{2}=0.13\right)$.

\section{Discussion}

Our long-term radiotracking study of many individuals followed continuously throughout the year support the importance of considering a combination of different spatio-temporal scales and individual-level processes when studying home range behaviour. Our most important results indicated that among individuals heterogeneity and withinindividual consistency in behaviours played the most important role in shaping home range characteristics. Additionally, but with low explanatory powers of the models, we found that (1) external and internal determinants may simultaneously affect the home range behaviour of owners and that (2) their relative effects differ among different spatio-temporal scales. In particular: (1) the effect of the different phases of the biological cycle became evident at the level of movement patterns, and (2) both external (i.e. habitat structure and composition) and internal (i.e. sex and health state) factors partially explained the observed home range behaviour in terms of space use, movement patterns and rhythms of activity.

If such results have also drawn attention to the occurrence of multi-level factors and processes affecting home range behaviour, our main and probably unexpected result was the detection of a scenario that is profoundly dominated by the individual and its intrinsic characteristics (as revealed by the ICC and $R^{2}$ values for owl ID random terms). Across the different spatial and temporal components of our study, we detected both inter-individual variations in home range behaviour (to a relatively variable degree, depending on the specific variable we were analysing) and intra-individual consistency in the way the owls behaved over the study period: both inter- and intra-individual effects largely contributed to shaping (1) home range and core area sizes and (2) movement patterns during different periods of the biological cycle. These findings are in agreement with those of recent studies that assessed intraspecific variations in home range behaviour (Saïd et al. 2009; Börger et al. 2006; van Beest et al. 2011), in which a considerable portion of the home range variance was partly ascribed to differences among individuals; i.e. every individual is a unique entity as a result of its own experience across the different life stages, as a nestling, fledgling, dispersing and floating juvenile as well as a more or less experienced breeder. Each individual is the result of a series of complex, reciprocal interactions between factors that can occur throughout an individual's lifetime and are responsible for the emergence of different personalities (Sasha et al. 2004; Stamps and Groothuis 2010). Thus, it makes sense to assume that heterogeneity in home range behaviours, which at the individual-level, could be consistent over time (i.e. year-round) and may be not fully captured by certain fundamental biological traits (e.g. sex, age or physiological condition), could be partly explained by different personality types within the same population (Both et al. 2005). Although different home range behaviours may also be a consequence of changing environmental conditions and habitat heterogeneity (e.g. Fraser et al. 2001; Delgado and Penteriani 2008; Stamps and Groothuis 2010), when local conditions and resources are stable over time and homogeneously distributed in space, individual personalities may become more evident and may thus be one of the most crucial factors in determining the behavioural patterns of a population.

With respect to temporal implications of the observed home range behaviour, as expected, the owls showed seasonal variations in their movement patterns at the home range scale. Males travelled longer distances during the incubation and nestling periods, probably because they 
play a key role during the beginning of the breeding period, as they are responsible for female feeding and breeding territory defence (Penteriani and Delgado 2008b). These two activities may require males to continuously move back and forth from and to the nest site, crossing extensive portions of their home range and core area(s) to (1) prevent intruders from approaching their breeding areas, (2) perform territorial displays (eagle owl territorial displays do not end after the egg-laying period; Delgado and Penteriani 2007), and (3) search for food. It is well known that reproduction is energetically expensive for both mates, but from a movement perspective, males have to sustain more continuous activities, travel over longer distances and undergo higher rates of movement. Additionally, most likely due to offspring-parent interactions throughout the F/PFD period, both males and females exhibited decreased displacements during this phase of the breeding cycle. From fledging, when sibling movement skills are still limited, to the post-fledging dependence period, when the distances travelled by juveniles from the nest increase, the explorations of the natal area by young birds are mainly limited to near the nest (Delgado et al. 2009). At this stage, because siblings are still under the nearly continuous control of their parents (females at least; Delgado et al. 2009), they might be forced to move shorter distances than in other periods.

Despite these temporal variations in movement patterns, eagle owl home range behaviour as a whole did not vary across the biological cycle, suggesting extremely stable home ranges. Constant and well-established home ranges may be the result of systematic movement strategies (Fortin 2002), such as those shown by the owners of a breeding site (Delgado et al. 2009), that work when a priori information is available and allow optimal coverage of a given area based on relatively fixed and controlled plans. In fact, sedentary species are expected to exhibit strong interactions between individual behaviours and their spatial context (Börger et al. 2006). However, although we did not take into account the possible effect of conspecific density in the present study, the extremely high density of breeders in our study area, combined with the high territoriality of males (Penteriani and Delgado 2008b), could have strongly limited conspicuous home range expansions/contractions. Each eagle owl home range seems to have a well-determined location and size throughout the year. Under this framework, considerable alterations of home range boundaries among periods are not allowed, whereas withinboundary movements (e.g. total distance and step length) were somewhat variable over the temporal scale examined in this study.

Home ranges may represent an invisible link between the movements of individuals and the distribution of the resources necessary to survive and reproduce (Börger et al.
2008). Hence, if such resources are heterogeneously distributed among different habitat types, or if their occurrence is influenced by landscape structure, then landscape properties can affect habitat selection and use, which, in turn, can modify home range spatial patterns (Pasinelli 2000; Indermaur et al. 2009). Our findings indicate that most of the variation in home range and core area size is principally determined by edge density, which is a proxy of landscape heterogeneity and fragmentation. As previously observed (e.g. Kie et al. 2002; Saïd and Servanty 2005), the dimensions of home ranges may be negatively correlated with the density of edges; i.e. most complex landscape matrices determine smaller home ranges. Higher densities of edges have the potential to aggregate different patch types in a reduced space (Tufto et al. 1996; Revilla et al. 2004), consequently determining a more clustered distribution of basic resources. As an end result, such crowded resources can reduce individual rates of movement and, thus, home range sizes. Additionally, edge density has been considered to be a good predictor of the distribution of areas suitable for the reproduction of another Mediterranean rabbit-specialist species, the Iberian lynx (Lynx pardinus), as increased edge density favours rabbit abundance (Fernández et al. 2003). Specifically, the structure of edges between shrubs and open areas allows rabbits to optimise their spatial behaviour and to easily access feeding and refuge patches (Lombardi et al. 2003, 2007). Because of the dependence of rabbits on this combination of edges, shrubs and open patches, it is not surprising that we also found a correlation between certain components of eagle owl movement patterns (i.e. turning angle) and rhythms of activity (i.e. movement rates) with landscape structure and composition. Thus, as predicted, the spatial heterogeneity of nesting site surroundings can affect the characteristics of a home range, which reveals individual decisions at this spatial scale (Hinsley et al. 1995; Knick and Rotenberry 1995). In fact, both the quality and the structure of habitats may engender diverse costs and benefits and, consequently, cause conspicuous behavioural differences (Diffendorfer et al. 1995), with landscapes being frequently highlighted as major factors driving animal movement patterns (e.g. Nathan et al. 2008; Delgado et al. 2010).

Given the general importance attributed to prey availability and distribution in shaping the behaviours of predators (e.g. Marquiss and Newton 1981; Selås and Rafoss 1999; Fernández et al. 2009), we expected that owl home range behaviour would also be highly responsive to rabbit abundance, with the owls varying their space use behaviour according to prey density. In contrast to our expectations, rabbit availability did not appear to affect home range behaviour at any spatio-temporal scale. The absence of a prey abundance effect on eagle owl home range behaviour 
could be due to the fact that prey does not generally represent a limiting factor in our population: our rabbit latrine count indicated high availability and abundance of rabbits inside the owl home range boundaries. For example, rabbit densities (1.0-4.6 individuals $\mathrm{ha}^{-1}$ ) lower than those recorded in our study area have been suggested to be suitable to support successful reproduction of Iberian lynxes, which do not alter their space use behaviour at this threshold of density (Palomares et al. 2001). Although heterogeneous patterns of prey spatial distributions are common in most natural scenarios (Bell 1991), rabbit management and frequent releases inside our study area could have created extremely favourable and steady trophic conditions. This peculiar abundance of a homogeneously distributed prey may also be reflected in the persistence of an extremely saturated eagle owl population, with a breeding density reaching approximately 40 breeding territories $100 \mathrm{~km}^{-2}$ with a mean NND of approx. $1 \mathrm{~km}$ (mean \pm SD: $982 \pm 491 \mathrm{~m}$, range 250-2,729 m; Mora et al. 2010). Under these circumstances, we can hypothesise that (1) prey density has reached a threshold that exceeds the eagle owls' pro capita needs everywhere in our study area, including during the most constraining periods (i.e. feeding of large broods) and, because of this peculiar ecological scenario, and (2) individuals do not need to alter their behaviour to confront seasonal environmental heterogeneity (e.g. Ferguson et al. 1999) or prey fluctuations (Delibes-Mateos et al. 2008). However, we should note that, although food appears to be abundant and homogeneously distributed throughout the entire study area, our results also confirmed one of the anticipated patterns, i.e. that individual variation in reproduction may be related to the intrinsic properties of home ranges, such as resource (i.e. prey) abundance. In fact, higher fecundities were associated with higher rabbit abundance in the eagle owl diet. This result means that, under a scenario in which prey abundance is high in every nesting site and breeding pairs may reproduce successfully every year, certain home ranges particularly rich in rabbits allow the extremely high fecundity rates (i.e. 3-4 fledglings per nest per year) that eagle owls mainly exhibit when their range of distribution overlaps with that of rabbits (Delibes and Hiraldo 1981).

Despite the strong individual signature regarding home range behaviour, sex and health state represented two additional factors that contributed to shaping home range behaviour, which was in agreement with our predictions. First, sex-dependent tasks have the potential to affect movement decisions at both daily (e.g. rhythm of activities) and seasonal (e.g. movement patterns) temporal scales and, thus, contribute to differentiating the patterns of space use of males and females that we observed at the larger (overall) temporal scale. In addition, male home range behaviour may also reflect social constraints because of strong male territoriality (Penteriani et al. 2007a). For example, males exhibited smaller home ranges with a simpler internal structure in which core areas were smaller in size than was seen for females (which are allowed to intrude into the territories of neighbouring pairs with less conflict; Penteriani et al. 2007a). Because the home ranges of neighbouring owls in our population may overlap and are very close to each other (V. Penteriani, M.M. Delgado and L. Campioni, in preparation), the movements of males outside their own home range boundaries can lead to risky and dangerous encounters with other males, which can show extremely aggressive behaviour (Penteriani et al. 2007a). In contrast, the intrusion of a new female can represent the possibility of occasional bigamy (Dalbeck et al. 1998; V. Penteriani and M.M. Delgado, unpublished data), and eagle owl females generally show low levels of aggression towards other females approaching their nesting site (Penteriani et al. 2007a). Finally, home range internal structure was related to differences in the state of individuals, with healthier owners being associated with a simpler internal home range structure. This relationship could suggest, for example, that the existence of fewer core areas and smaller distances between breeding and foraging sites may reduce movements and, consequently, minimise daily energetic expenditures allocated to unprofitable and costly activities (e.g. McNab 1963; Schoener 1968; Bell 1991).

Because of the plurality of behavioural and ecological processes simultaneously involved in the individual behavioural response to temporal and spatial variations of internal and external factors, home range behaviour remains one of the most appealing and challenging processes to study in the field of animal ecology. Our results provide strong empirical evidence of the crucial relevance of individual-level processes over time and space, suggesting that variation of space use patterns within the same population can be the fingerprint of individual- and sitespecific behavioural and ecological dynamics taking place under unique local conditions. In a time associated with great environmental changes, in which the majority of attention is currently focused on the effects of habitat heterogeneity, alterations and/or fragmentation, we emphasised the importance of still addressing our interest on favourable and homogeneous environmental conditions. Stable environments may provide a fertile context in which to re-direct our interest toward exploring and analytically capturing intraspecific differences in behaviour, which, to date, remain difficult to recognise as personality types, even though inter-individual variation in behaviour is often distributed in a non-random manner, suggesting that it is likely to have consistent ecological and evolutionary consequences (Sasha et al. 2004). 
Acknowledgments We wish to thank P. Bartolommei, C. Bettega, B. Fant and C. Maggio for their help with fieldwork. We also are grateful to R. Alonso for his help in aging eagle owls. We thank Markku Orell and two anonymous referees for their helpful comments and improvements of the manuscript. The work was funded by two research projects of the Spanish Ministry of Science and Innovation (CGL2004-02780/BOS and CGL2008-02871/BOS; with FEDER co-financing), the Ministry of Education and Science-C.S.I.C. (Proyectos Intramurales Especiales, DG-2606-PC), the Junta of Andalucía (Excellence Project, RNM5090), and LICOR43. During this work, L.C. was supported by the JAE pre-doctoral grant from the C.S.I.C., V.P. received a grant from the Spanish Secretaría General de Universidades, Ministry of Education (Salvador de Madariaga Program), and M.M.D. was recipient of a postdoctoral fellowship from the Spanish Ministry of Science and Innovation, and a post-doctoral grant from the Finnish Academy n ${ }^{\circ} 140367$. Owls were trapped and marked under the Junta de Andalucía-Consejería de Medio Ambiente permit nos. SCFFSAFR/GGG RS-260/02 and SCFFS-AFR/CMM RS-1904/02.

\section{References}

Aebischer NJ, Robertson PA, Kenward RE (1993) Compositional analysis of habitat use from animal radio tracking data. Ecology 74:1313-1325. doi:10.2307/1940062

Anderson DP, Forester JD, Turner MG, Frair JL, Merrill EH, Fortin D, Mao JS, Boyce MS (2005) Factors influencing female home range sizes in elk (Cervus elaphus) in North American landscapes. Lands Ecol 20:257-271. doi:10.1007/s10980-0050062-8

Bates DM, Sarkar D (2007) lme4: linear mixed-effects models using S4 classes, R package version 0.99875-6

Bell WJ (1991) Searching behaviour: the behavioural ecology of finding resources. Chapman and Hall, London

Börger L, Franconi N, Ferretti N, Meschi F, De Michele G, Gantz A, Coulson T (2006) An integrated approach to identify spatiotemporal and individual-level determinants of animal home range size. Am Nat 168:471-485

Börger L, Danziel BD, Fryxell JM (2008) Are there general mechanisms of animal home range behaviour? A review and prospects for future research. Ecol Lett 11:637-650. doi: 10.1111/j.1461-0248.2008.01182.x

Both C, Dingemanse NJ, Drent PJ, Tinbergen JM (2005) Pairs of extreme avian personalities have highest reproductive success. J Anim Ecol 74:667-674. doi:10.1111/j.1365-2656.2005. 00962.x

Burnham KP, Anderson DR (2002) Model selection and multi-model inference: a practical information-theoretic approach, 2nd edn. Springer, New York

Cagnacci F, Boitani L, Powell RA, Boyce MS (2010) Challenges and opportunities of using GPS-based location data in animal ecology. Philos Trans R Soc Lond B 365:2153-2312. ISSN 0962-8436

Calenge C (2006) The package adehabitat for the R software: a tool for the analysis of space and habitat use by animals. Ecol Mod 197:516-519. doi:10.1016/j.ecolmodel.2006.03.017

Costa FJV, Macedo RH (2006) Coccidian oocyst parasitism in the blueblack grassiquit: influence on secondary sex ornaments and body condition. Anim Behav 70:1401-1409. doi:10.1016/j.anbehav. 2005.03.024

Cramp S, Simmons KEL (1977-1994) Birds of the western Palearctic, Oxford University Press, Oxford

Dalbeck L, Bergerhausen W, Krishner O (1998) Telemetriestudie zur Orts- und Partnertreue beim Uhu Bubo bubo. Vogelwelt 119:37-344
Darwin C (1861) On the origin of species by means natural selection, 3rd edn. Murray, London

De Solla SR, Bonduriansky R, Brooks RJ (1999) Eliminating autocorrelation reduces biological relevance of home range estimates. J Anim Ecol 68:221-234. doi:10.1046/j.1365-2656.1999. 00279.x

Delgado MM, Penteriani V (2007) Vocal behaviour and neighbour spatial arrangement during vocal displays in eagle owl. J Zool 271:3-10. doi:10.1111/j.1469-7998.2006.00205.x

Delgado MM, Penteriani V (2008) Behavioral states help translate dispersal movements into spatial distribution patterns of floaters. Am Nat 172:475-485. doi:10.1086/590964

Delgado MM, Penteriani V, Nams O (2009) How fledglings explore surroundings from fledging to dispersal. A case study with eagle owls Bubo bubo. Ardea 97:7-15. doi:10.5253/078.097.0102

Delgado MM, Penteriani V, Revilla E, Nams VO (2010) The effect of phenotypic traits and external cues on natal dispersal movements. J Anim Ecol 79:620-632. doi:10.1111/j.1365-2656.2009. 01655.x

Delibes M, Hiraldo F (1981) The rabbit as prey in the Iberian Mediterranean ecosystem. In: Myers K, Innes CD (eds) Proceedings of the World Lagomorph Conference 1979, University of Guelph, Ontario, pp 614-622

Delibes-Mateos M, Redpath SM, Angulo E, Ferreras P, Villafuerte R (2007) Rabbits as a keystone species in southern Europe. Biol Conserv 137:149-156. doi:10.1016/j.biocon.2007.01.024

Delibes-Mateos M, Ferreras P, Villafuerte R (2008) Rabbit populations and game management: the situation after 15 years of rabbit haemorrhagic disease in central-southern Spain. Biodiv Conserv 17:559-574. doi:10.1007/s10531-007-9272-5

Diffendorfer JE, Gaines MS, Holt RD (1995) Habitat fragmentation and movements of three small mammals (Sigmodon, Microtus, and Peromyscus). Ecology 76:827-839. doi:10.2307/1939348

Donázar JA, Ceballos O (1989) Selective predation by eagle owls Bubo bubo on rabbits Oryctolagus cuniculus: age and sex preferences. Ornis Scand 20:117-122

Donovan TM, Thompson FR, Faaborg J, Probst JR (1995) Reproductive success of migratory birds in habitat sources and sinks. Conserv Biol 9:1380-1395. doi:10.1046/j.1523-1739.1995. 09061380.x

Elkie P, Rempel R, Carr A (1999) Patch analyst user's manual: a tool for quantifying landscape structure. Ontario Ministry of Natural Resources Northwest Science and Technology. http://flash.lakeh eadu.ca/_rrempe/patch/

Ferguson SH, Taylor MK, Born EW, Rosing-Asvid A, Messier F (1999) Determinants of home range size for polar bears (Ursus maritimus). Ecol Lett 2:311-318. doi:10.1046/j.1461-0248.1999. 00090.x

Fernández N (2005) Spatial patterns in European rabbit abundance after a population collapse. Landsc Ecol 20:897-910. doi: 10.1007/s10980-004-3976-7

Fernández N, Delibes M, Palomares F, Mladenoff DJ (2003) Identifying breeding habitat for the Iberian lynx: inferences from a fine-scale spatial analysis. Ecol Appl 13:1310-1324. doi: $10.1890 / 02-5081$

Fernández M, Oria J, Sánchez R, Gonzalez LM, Margalida A (2009) Space use of adult Spanish Imperial Eagles Aquila adalberti. Acta Ornithol 44:17-26. doi:10.3161/000164509X464849

Fortin D (2002) Optimal searching behaviour: the value of sampling information. Ecol Mod 153:279-290. doi:10.1016/S0304-3800 (02)00031-5

Fraser DF, Gilliam JF, Daley MJ, Le AN, Skalski GT (2001) Explaining leptokurtic movement distributions: intrapopulation variation in boldness and exploration. Am Nat 158:124-135

González LM, Oria J, Sánchez R, Margalida A, Aranda A, Prada L, Caldera J, Molina JI (2008) Status and habitat changes in the 
endangered Spanish Imperial Eagle Aquila adalberti population during 1974-2004: implications for its recovery. Bird Conserv Int 18:242-259. doi:10.1017/S0959270908000245

Griffiths R, Double MC, Orr K, Dawson RJG (1998) A DNA test to sex most birds. Mol Ecol 7:1071-1075. doi:10.1046/j.1365-294x. 1998.00389.x

Hayne DW (1949) Calculation of home range size. J Mammal 30:1-18

Hays G (2008) Virtual issue: biotelemetry and biologging. J Anim Ecol 77. http://www.journalofanimalecology.org/view/0/virtualissue1.html

Hinsley SA, Bellamy PE, Newton I, Sparks TH (1995) Habitat and landscape factors influencing the presence of individual breeding bird species in woodland fragments. J Avian Biol 26:94-104

Hooge PN, Eichenlaub B (2000) Animal movement extension to ArcView, version 2.0.3. Biological Science Centre, US Geological Survey, Anchorage, Alaska. http://www.absc.usgs.gov/glba/ gistools/index.htm

Horne JS, Garton EO, Rachlowa JL (2008) A synoptic model of animal space use: simultaneous estimation of home range, habitat selection, and inter/intra-specific relationships. Ecol Mod 214:338-348. doi:10.1016/j.ecolmodel.2008.02.042

Hothorn T, Bretz F, Westfall P (2009) multcomp: simultaneous inference for general linear hypotheses. http://CRAN.R-project.org/ package $=$ multcomp. $\mathrm{R}$ package version 1.0-7

Indermaur L, Gehring M, Wehrle W, Tockner K, Naef-Daenzer B (2009) Behavior-based scale definition for detecting individual space use: requirements of two amphibians. Am Nat 173:60-71

Kernohan BJ, Gitzen RA, Millspaugh JJ (2001) Analysis of animal space use and movements. In: Millspaugh JJ, Marzluff JM (eds) Radiotracking and animal populations. Academic, San Diego, pp 125-166

Kie JG, Bowyer RT, Nicholson MC, Boroski BB, Loft ER (2002) Landscape heterogeneity at differing scales: effects on spatial distribution of mule deer. Ecology 83:530-544

Kie JG, Matthiopoulos J, Fieberg J, Powell RA, Cagnacci F, Mitchell MS, Gaillard J-M, Moorcroft PR (2010) The home-range concept: are traditional estimators still relevant with modern telemetry technology? Phil Trans R Soc Lond B 365:2221-2231. doi:10.1098/rstb.2010.0093

Knick ST, Rotenberry JT (1995) Landscape characteristics of fragmented shrubsteppe habitats and breeding passerine birds. Conserv Biol 9:1059-1071

Lombardi L, Fernández N, Moreno S, Villafuerte R (2003) Habitatrelated differences in rabbit (Oryctolagus cuniculus) abundance, distribution, and activity. J Mammal 84:26-36. doi:10.1644/ 1545-1542(2003)084<0026:HRDIRO $>2.0 . \mathrm{CO} ; 2$

Lombardi L, Fernández N, Moreno S (2007) Habitat use and spatial behaviour in the European rabbit in three mediterranean environments. Bas Appl Ecol 8:453-463. doi:10.1016/j.baae.2006.09.004

Lourenço R (2006) The food habits of Eurasian eagle-owls in Southern Portugal. J Raptor Res 40:297-300. doi:10.3356/0892-1016 (2006)40[297:TFHOEE]2.0.CO;2

MacDonald DW, Barret P (1993) Mammals of Britain and Europe. Harper-Collins, London

Marquiss M, Newton I (1981) A radio-tracking study of the ranging behaviour and dispersion of European Sparrow hawks Accipiter nisus. J Anim Ecol 51:111-133

Martínez CJA, Zuberogoitia AI, Alonso MR (2002) Rapaces nocturnas. Guía para la determinación de la edad y el sexo en las estrigiformes ibéricas, 1 st edn. Monticala, Madrid

McLoughlin PD, Ferguson SH (2000) A hierarchical pattern of limiting factors helps explain variation in home range size. Ecoscience 7:123-130

McNab BK (1963) Bioenergetic and the determination of home range size. Am Nat 97:133-140
Mora O, Delgado MM, Penteriani V (2010) Secondary sex ratio in Eurasian Eagle-owls: early-breeding females produce more daughters. J Raptor Res 44:62-65

Morales JM, Moorcroft PR, Matthiopoulos J, Frair JL, Kie JG, Powell RA, Merrill EH, Haydon DT (2010) Building the bridge between animal movement and population dynamics. Phil Trans R Soc Lond B 365:2289-2301. doi:10.1098/rstb.2010.0082

Nathan R, Getz WO, Revilla E, Holyoak M, Kadmon R, Saltz D, Smouse PE (2008) A movement ecology paradigm for unifying organism movement research. Proc Natl Acad Sci USA 105:19052-19059. doi:10.1073/pnas.0800375105

Palma L, Beja P, Rodrigues M (1999) The use of sighting data to analyse Iberian lynx habitat and distribution. J Appl Ecol $36: 812-824$

Palomares F (2001a) Comparison of 3 methods to estimate rabbit abundance in a Mediterranean environment. Wildl Soc Bull 29:578-585

Palomares F (2001b) Vegetation structure and prey abundance requirements of the Iberian lynx: implications for the design of reserves and corridors. J Appl Ecol 38:9-18. doi:10.1046/j. 1365-2664.2001.00565.x

Palomares F, Dlibes M, Revilla E, Calzada J, Fedriani JF (2001) Spatial ecology of Iberian lynx and abundance of European rabbits in southwestern Spain. Wildl Monogr 148:1-36

Pasinelli G (2000) Oaks (Quercus sp.) and only oaks? Relations between habitat structure and home range size of the middle spotted woodpecker (Dendrocopos medius). Biol Conserv 93:227-235. doi:10.1016/S0006-3207(99)00137-8

Penteriani V, Delgado MM (2008a) Brood-switching in eagle owl Bubo bubo fledglings. Ibis 150:816-819. doi:10.1111/j.1474-919X. 2008.00831.x

Penteriani V, Delgado MM (2008b) Owls may use faeces and prey feathers to signal current reproduction. PLoS One 3(8):e3014. doi:10.1371/journal.pone.0003014

Penteriani V, Delgado MM, Maggio C, Aradis A, Sergio F (2005) Development of chicks and pre-dispersal behaviour of young in the eagle owl Bubo bubo. Ibis 147:155-168. doi:10.1111/j.1474919x.2004.00381.x

Penteriani V, Alonso-Alvarez C, Delgado MM, Sergio F (2007a) The importance of visual cues for nocturnal species: eagle owls signal by badge brightness. Behav Ecol 18:143-147. doi: 10.1093/beheco/arl060

Penteriani V, Delgado MM, Alonso-Alvarez C, Viqueira Pina N, Sergio F, Bartolommei P (2007b) The importance of visual cues for nocturnal species: eagle Owl fledglings signal with white mouth feathers. Ethology 113:934-943. doi:10.1111/j.1439-0310.2007. 01414.x

Penteriani V, Delgado MM, Maggio C, Alonso-Alvarez C, Holloway GJ (2008) Owls and rabbits: selective predation against substandard individuals by a sit-and-wait predator. J Avian Biol 39:215-221. doi:10.1111/j.0908-8857.2008.04280.x

Penteriani V, Delgado MM, Campioni L, Lourenço R (2010) Moonlight makes owls more chatty. PLoS One 5(1):e8696. doi: 10.1371/journal.pone.0008696

Pinheiro JC, Bates DM (2004) Mixed-effects Models in S and S-PLUS. Springer, New York

Pinheiro JC, Bates DM, DebRoy S, Sarkar D, R Core team (2009) nlme: linear and nonlinear mixed effects models. $\mathrm{R}$ package version 3.1-90

R Development Core Team (2009) R: a language and environment for statistical computing. R Foundation for Statistical Computing, Vienna

Revilla E, Wiegand T, Palomares F, Ferreras P, Delibes M (2004) Effects of matrix heterogeneity on animal dispersal: from individual behavior to metapopulation-level parameters. Am Nat 164:130-153 
Rivrud IM, Loe LE, Mysterud A (2010) How does local weather predict red deer home range size at different temporal scale? J Anim Ecol 79:1280-1295. doi:10.1111/j.1365-2656.2010.01731.x

Said S, Servanty S (2005) The influence of landscape structureon female roe deer home-range size. Landsc Ecol 20:1003-1012

Saïd S, Gaillard JM, Widmer O, Débias F, Bourgoin G (2009) What shape intra- specific variation in home range size? A case study of female roe deer. Oikos 118:1299-1306. doi:10.1111/j.1600-0706. 2009.17346.X

Sasha RX, Houston AI, McNamara JM (2004) The behavioural ecology of personality: consistent individual differences from adaptive perspective. Ecol Lett 7:734-739. doi:10.1111/j.14610248.2004.00618.x

Schoener TW (1968) Size and feeding territories among birds. Ecology 49:123-141

Seaman DE, Powell RA (1996) An evaluation of the accuracy of Kernel density estimators for home range analysis. Ecology 77:2075-2085

Seaman DE, Millspaugh JJ, Kernohan BJ, Brundige GC, Raedeke KJ, Gitzen RA (1999) Effects of sample size on kernel home range estimates. J Wildl Manag 63:739-747

Selås V, Rafoss T (1999) Ranging behaviour and foraging habitats of breeding Sparrow hawks Accipiter nisus in a continuous forested area in Norway. Ibis 141:269-276. doi:10.1111/j.1474-919X. 1999.tb07549.x

Silverman BW (1986) Density estimation for statistics and data analysis. Monographs on statistics and applied probability. Chapman and Hall, London
Smouse PE, Focardi S, Moorcroft PR, Kie JG, Forester JD, Morales JM (2010) Stochastic modelling of animal movement. Phil Trans R Soc Lond B 365:2201-2211. doi:10.1098/rstb.2010.0078

Stamps JA, Groothuis GG (2010) Developmental perspectives on personality: implications for ecological and evolutionary studies of individual differences. Phil Trans R Soc Lond B 365: 4029-4041. doi:10.1098/rstb.2010.0218

Tufto J, Andersen R, Linnell JDC (1996) Habitat use and ecological correlates of home range size in a small cervid: the roe deer. J Anim Ecol 65:715-724

van Beest FM, Rivrud IM, Loe LE, Milner JM, Mysterud A (2011) What determines variation in home range sizes across spatiotemporal scales in a large browsing herbivore? J Anim Ecol 80:771-785. doi:10.1111/j.1365-2656.2011.01829.x

Worton BJ (1989) Kernel methods for estimating the utilization distribution in home range studies. Ecology 70:164-168. doi: $10.2307 / 1938423$

Zheng B, Agresti A (2000) Summarizing the predicted power of a generalized linear model. Stat Med 19:1771-1781. doi:10.1002/ 1097-0258(20000715)19:13<1771:AID-SIM485>3.0.CO;2-P

Zuur AF, Ieno EN, Walker NJ, Saveliev AA, Smith GM (2009) Mixed effects models and extensions in ecology with $\mathrm{R}$. Springer, New York 